\title{
Second-Order Phase Transitions in Non-Magnetic Wurtzite ZnO and Magnetic Doped with Co
}

\author{
H.W. KunERT* AND P. NiYONGABO
}

Department of Physics, University of Pretoria, Pretoria 0002, South Africa

We investigate the second-order phase transitions in non-magnetic wurtzite $\mathrm{ZnO}$ and magnetic doped with cobalt. Using reformulated Landau-Lifshitz theory of second-order phase transitions and our computer program, we have found all possible lower space group symmetries of $\mathrm{ZnO}$ and $\mathrm{ZnO}$ doped with $\mathrm{Co}$ as well as symmetries of vibration modes which may cause structural phase transitions. We interpret the Raman phonon modes of magnetic doped $\mathrm{ZnO}$ according to corepresentations of the magnetic space group $P 6_{3}^{\prime} m^{\prime} c$ ( $\mathrm{ZnO}$ magnetic). Some experimental techniques like X-ray diffraction, reflectivities and Raman spectroscopies can verify our theoretical results.

DOI: 10.12693 /APhysPolA.127.400

PACS: 75.50.Pp

\section{Introduction}

The $\mathrm{ZnO}$ undergoes several structural transitions upon applications of temperature and pressure. When it is doped with magnetic elements such as Co during synthesis, layers of the magnetic elements place themselves between two sublattices of $\mathrm{ZnO}$ and $\mathrm{O}$, and the whole crystal becomes magnetic. Consequently, the symmetry of $\mathrm{ZnO}\left(C_{6 v}^{4}\right)$ changes because an extra time reversal symmetry operator (which reverses magnetic moments $(\bar{\mu} \rightarrow-\bar{\mu})$ of dopants and brings $t$ to $-t$ and i to $-\mathrm{i})$ is added to the symmetry operators of $C_{6 v}^{4}\left(P 6_{3} m c\right)$ resulting in magnetic group; $C_{6 v}^{4}\left(C_{6}^{6}\right) \Rightarrow M\left(P 6_{3^{\prime}} m^{\prime} c\right)$. Our computerized method yields all subgroups of the initial space group $G_{0}$. The reformulated Landau-Lifshitz and Lyubarskii (LLL) [1, 2] method for magnetic space group restricts the number of possible magnetic space group. The allowed magnetic subgroups are obtained from active physical irreducible corepresentations of the initial magnetic space group. Therefore, in magnetic crystals the states of particles like electrons, holes, etc. and quasiparticles like phonons, magnons, etc. are classified according to irreducible representations (irreps) of magnetic groups called corepresentations (coreps) [3].

In Sect. 2 we have outlined the group theoretical criteria for second-order phase transitions (S.O.Ph.Tr) in non-magnetic crystals in terms of irreps and their symmetrized and antisymmetrized Kronecker products (KPs): $\{D\}_{2}$ and $[D]_{3}[4]$. Cracknell has reformulated the $\mathrm{L}-\mathrm{L}$ criteria for magnetic transitions in terms of symmetrized and antisymmetrized coreps; $\{C D\}_{2}$ and $[C D]_{3}$ and applied to compounds of magnetic group $m 3^{\prime}$. Here we investigate S.O.Ph.Tr in both compounds using standard L-L theory and modified for magnetic $\mathrm{ZnO}$.

\section{Second-order phase transitions in non-magnetic $\mathrm{ZnO}$}

According to Landau-Lifshitz and Lyubarskii (LLL) $[1,2]$ theory the thermodynamic potential

\footnotetext{
* corresponding author; e-mail: Herbert.Kunert@up.ac.za
}

for non-magnetic crystals extended up to fifth order is invariant with respect to unitary space group operators of the initial unitary group $G_{0}$. Consequently, several criteria for transitions have been discussed by many authors in terms of irreps of unitary space groups [5]. According to LLL theory the irreps of $G_{1}$ (space group after transitions) and $G_{0}$ (space group before transitions) must satisfy several criteria for transitions between phases. These are:

- The space group $G_{1}$ of the crystal after transition is a subgroup of $G_{0}$ before phase transition.

- Symmetrized cube of $D \rightarrow[D]_{3}$ involved in transition must not contain identity irrep of $G_{0}$ usually denoted by $\Gamma_{1}$ or $\Gamma_{1+}$ or $A_{1+}$.

- Antisymmetrized square of $D \rightarrow\{D\}_{2}$ must not contain any representation which has any component of a vector representation $(V R)$ as its base $(x, y, z)$.

- The irrep $D$ must be compatible with the identity irrep of $G_{1}$.

- The irrep $D$ must be real and therefore must satisfy the reality test [6].

The irreps satisfying the above criteria are known as active irreps and may cause S.O.Ph.Tr. The above criteria are concerned with unitary space group and their unitary irreps. Using the above criteria we have determined possible space subgroups of $\mathrm{ZnO}\left(C_{6 v}^{4}\right)$ and the active irreps of initial space group responsible for transitions (see Tables I and II). The Raman spectroscopy can identify which subgroup is involved after transition.

TABLE I

Subgroups $\mathrm{H}$ of $C_{6 v}^{4}$ and their symmetry operators using CDML labelling [8].

\begin{tabular}{|c|c|c|c|c|c|c|c|c|c|c|c|}
\hline$c_{6 v}^{4}(P 63 m c, 186)$ & 12.1 & 3 & 4.1 & 5 & 6.1 & 19 & 20.1 & 21 & 22.1 & 23 & 24.1 \\
\hline$C_{6}^{6}\left(P 6_{3} ; 173\right)$ & 12.1 & 3 & 4.1 & 5 & 6.1 & & & & & & \\
\hline$C_{3 v}^{4}(P 31 c ; 159)$ & 1 & 3 & & 5 & & & 20.1 & & 22.1 & & 24.1 \\
\hline$C_{3 v}^{1}(P 3 m 1 ; 156)$ & 1 & 3 & & 5 & & 19 & & 21 & & 23 & \\
\hline$C_{3}^{4}(R 3 ; 146)$ & 1 & 3 & & 5 & & & & & & & \\
\hline$C_{3}^{1}(P 3 ; 143)$ & 1 & 3 & & 5 & & & & & & & \\
\hline
\end{tabular}


TABLE II

Active irreducible representations of initial space group $\left(G_{0}=C_{6 v}^{4}\right)$ that may cause S.Or.Ph.Trs by active modes of high symmetry points $\Gamma, A, K, H, M$ and $L$ to lower space group symmetry $G$.

\begin{tabular}{l|l}
\hline \hline $\begin{array}{l}\text { Symmetry operators of the group } \\
\text { of high symmetry: } G_{0}\left(C_{6 v}\right)\end{array}$ & Subgroups of $G_{0}$ \\
\hline$C_{6 v}^{4}(\Gamma: 1,2.1,3,4.1,5,6.1,19,20.1,21,22.1,23 ., 24.1)$ & $C_{6 v}^{4} \stackrel{\Gamma_{2}}{\longrightarrow} C_{6}^{6}(1,2.1,3,4.1,5,6.1)$ \\
& $C_{6 v}^{4} \stackrel{\Gamma_{2}, 3,4}{\longrightarrow} C_{3}^{1}$ and $C_{3}^{4}(1,3,5)$ \\
& $C_{6 v}^{4} \stackrel{\Gamma_{3}}{\longrightarrow} C_{3 V}^{4}(1,3,5,20.1,22.1,24.1)$ \\
& $C_{6 v}^{4} \stackrel{\Gamma_{4}}{\longrightarrow} C_{3 V}^{1}(1,3,5,19,21,23)$ \\
& $C_{6 v}^{4} \stackrel{A_{1} \oplus A_{1}^{*}}{\longrightarrow} C_{3 v}^{1}(1,3,5,19,21,23)$ \\
$C_{6 v}^{4}(A: 1,2.1,3,4.1,5,6.1,19,20.1,21,22.1,23 ., 24.1)$ & $C_{6 v}^{4} \stackrel{A_{2} \oplus A_{2}^{*}}{\longrightarrow} C_{3}^{1}$ and $C_{3}^{4}(1,3,5)$ \\
& $C_{6 v}^{4} \stackrel{A_{5} \oplus A_{5}^{*}}{\longrightarrow} C_{3}^{1}$ and $C_{3}^{4}(1,3,5)$ \\
\hline$C_{6 v}^{4}(K: 1,3,5,20.1,22.1,24.1)$ & $C_{6 v}^{4} \stackrel{K_{2}}{\longrightarrow} C_{3}^{1}$ and $C_{3}^{4}(1,3,5)$ \\
\hline$C_{6 v}^{4}(H: 1,3,5,20.1,22.1,24.1)$ & $C_{6 v}^{4} \stackrel{H_{1} \oplus H_{1}^{*}}{\longrightarrow} C_{3}^{1}$ and $C_{3}^{4}(1,3,5)$ \\
\hline$C_{6 v}^{4}(L: 1,4.1,20.1,23)$ & $C_{6 v}^{4} \stackrel{L_{1} \oplus L_{1}^{*}}{\longrightarrow} C_{3 v}^{1}(1,23)$ \\
$C_{6 v}^{4}(M: 1,4.1,20.1,23)$ & $C_{6 v}^{4} \stackrel{M_{2}}{\longrightarrow} C_{6}^{6}(1,4.1)$ \\
& $C_{6 v}^{4} \stackrel{M_{3}}{\longrightarrow} C_{3 v}^{4}(1,20.1)$ \\
& $C_{6 v}^{4} \stackrel{M_{4}}{\longrightarrow} C_{3 v}^{1}(1,23)$ \\
\hline
\end{tabular}

\section{Magnetic phase transitions in $\mathrm{ZnO}$ doped with Co}

The main criterion for transition from original magnetic phase to a new magnetic phase is that the magnetic space group after transition must be a subgroup of the space group before transition. In Table III, we list the respective magnetic space subgroups and the phonon mode symmetries involved in the transitions using our computational methods. Similar to the procedure applied for non-magnetic $\mathrm{ZnO}$, the criteria for active coreps are: the symmetrized cube of coreps, $[C D]_{3}$ must not contain the identity corep $C D_{1}$ and the antisymmetrized square of coreps, $\{C D\}_{2}$ must not contain the corep to which the polar vector corep belongs. Using the Wigner theory [3] we have calculated coreps listed in Tables IV and $\mathrm{V}$.

TABLE III

Active irreducible corepresentations of initial magnetic space groups: $M_{0} \equiv C_{6 v}^{4}\left(C_{6}^{6}\right)\left(P 6_{3} m^{\prime} c^{\prime}\right), C_{6 v}^{4}\left(C_{3 v}^{4}\right)\left(P 6_{3}^{\prime} m^{\prime} c\right)$, $C_{6 v}^{4}\left(C_{3 v}^{1}\right)\left(P 6_{3}^{\prime} m c^{\prime}\right)$ that may cause S.Or.Ph. Trs by active modes of $C \Gamma, \mathrm{CA}$, to lower magnetic space group symmetry $M$.

\begin{tabular}{l|l}
\hline \hline Symmetry operators of the magnetic group of high symmetry: $M_{0}$ & Subgroups of the magnetic group of high symmetry: $M_{1}$ \\
\hline$C_{6 v}^{4}\left(C_{6}^{6}\right): P 6_{3} m^{\prime} c^{\prime}(1,2.1,3,4.1,5,6.1, \theta 19, \theta 20.1, \theta 21, \theta 22.1, \theta 23, \theta 24.1)$ & $P 6_{3} m^{\prime} c^{\prime} \stackrel{C \Gamma_{2}, C A_{2}}{\longrightarrow} C_{3 v}^{1}\left(C_{3}^{1}\right): P 3 m^{\prime} 1(1,3,5, \theta 19, \theta 21, \theta 23)$ \\
\hline$C_{6 v}^{4}\left(C_{3 v}^{1}\right): P 6_{3}^{\prime} m^{\prime} c(1,3,5,20.1,22.1,24.1, \theta 2.1, \theta 4.1, \theta 6.1, \theta 19, \theta 21, \theta 23)$ & $P 6_{3}^{\prime} m^{\prime} c \stackrel{C \Gamma_{2}, C A_{2}}{\longrightarrow} C_{6}^{6}\left(C_{3}^{1}\right): P 63^{\prime}(1,3,5, \theta 2.1, \theta 4.1, \theta 6.1)$ \\
\hline$C_{6 v}^{4}\left(C_{3 v}^{4}\right): P 6_{3}^{\prime} m c^{\prime}(1,3,5,19,21,23, \theta 2.1, \theta 4.1, \theta 6.1, \theta 20.1, \theta 22.1, \theta 24.1)$ & $P 6_{3}^{\prime} m c^{\prime} \stackrel{C \Gamma_{2}, C A_{2}}{\longrightarrow} C_{6}^{6}\left(C_{3}^{1}\right): P 63^{\prime}(1,3,5, \theta 2.1, \theta 4.1, \theta 6.1)$
\end{tabular}

Corepresentations for $M\left(P 6_{3}^{\prime} m^{\prime} c\right)$ magnetic space group,

where $\left(\begin{array}{ll}1 & 0 \\ 0 & 1\end{array}\right)=E$, and $\left(\begin{array}{cc}w & 0 \\ 0 & w^{*}\end{array}\right)=P$.

\begin{tabular}{c|cccccc}
\hline \hline & 1 & 2.1 & 3 & 4.1 & 5 & 6.1 \\
\hline$C \Gamma_{1}$ (a) & 1 & 1 & 1 & 1 & 1 & 1 \\
$C \Gamma_{2}(\mathrm{a})$ & 1 & -1 & 1 & -1 & 1 & -1 \\
$C \Gamma_{3,5}(\mathrm{c})$ & $\left(\begin{array}{cc}E & 0 \\
0 & E\end{array}\right)$ & $\left(\begin{array}{cc}P^{*} & 0 \\
0 & P^{*}\end{array}\right)$ & $\left(\begin{array}{cc}P & 0 \\
0 & P\end{array}\right)$ & $\left(\begin{array}{cc}E & 0 \\
0 & E\end{array}\right)$ & $\left(\begin{array}{cc}P^{*} & 0 \\
0 & P^{*}\end{array}\right)$ & $\left(\begin{array}{cc}P & 0 \\
0 & P\end{array}\right)$ \\
$C \Gamma_{4,6}(\mathrm{c})$ & $\left(\begin{array}{cc}E & 0 \\
0 & E\end{array}\right)$ & $\left(\begin{array}{cc}-P^{*} & 0 \\
0 & -P\end{array}\right)$ & $\left(\begin{array}{cc}P & 0 \\
0 & P\end{array}\right)$ & $\left(\begin{array}{cc}-E & 0 \\
0 & -E\end{array}\right)$ & $\left(\begin{array}{cc}P^{*} & 0 \\
0 & P^{*}\end{array}\right)$ & $\left(\begin{array}{cc}-P & 0 \\
0 & -P\end{array}\right)$ \\
$C A_{2}(\mathrm{a})$ & $-\mathrm{i}$ & 1 & $-\mathrm{i}$
\end{tabular}


Corepresentations continued for $M\left(P 6_{3}^{\prime} m^{\prime} c\right)$ magnetic space group.

\begin{tabular}{|c|c|c|c|c|c|c|}
\hline & $\theta 19$ & $\theta 20.1$ & $\theta 21$ & $\theta 22.1$ & $\theta 23$ & $\theta 24.1$ \\
\hline$C \Gamma_{1}(\mathrm{a})$ & 1 & 1 & 1 & 1 & 1 & 1 \\
\hline$C \Gamma_{2}(\mathrm{a})$ & 1 & -1 & 1 & -1 & 1 & -1 \\
\hline$C \Gamma_{3,5}$ (c) & $\left(\begin{array}{ll}0 & E \\
E & 0\end{array}\right)$ & $\left(\begin{array}{cc}0 & P^{*} \\
P & 0\end{array}\right)$ & $\begin{array}{cc}0 & P \\
P^{*} & 0\end{array}$ & $\left(\begin{array}{cc}0 & E \\
E & 0\end{array}\right)$ & $\begin{array}{ll}0 & P^{*} \\
P & 0\end{array}$ & $\left(\begin{array}{cc}0 & P \\
P^{*} & 0\end{array}\right)$ \\
\hline$C \Gamma_{4,6}(\mathrm{c})$ & $\left(\begin{array}{ll}0 & E \\
E & 0\end{array}\right)$ & $\begin{array}{cr}0 & -P \\
-P & 0\end{array}$ & $\begin{array}{cc}0 & P \\
P^{*} & 0\end{array}$ & $\begin{array}{cc}0 & -E \\
-E & 0\end{array}$ & $\begin{array}{cc}0 & P^{*} \\
P & 0\end{array}$ & $\begin{array}{cc}0 & -P \\
-P^{*} & 0\end{array}$ \\
\hline$C A_{2}(\mathrm{a})$ & 1 & $-\mathrm{i}$ & 1 & $-\mathrm{i}$ & 1 & $-\mathrm{i}$ \\
\hline
\end{tabular}

Using Tables IV and V and the equations: $\left\{C D_{i}\right\}_{2}=$ $\sum_{k} d_{2 i, k}^{a} C D_{k}$ and $\left[C D_{i}\right]_{3}=\sum_{k} d_{2 i, k}^{s} C D_{k}[4,7]$, we have determined the active coreps and these are $C A_{2}$ and $C \Gamma_{2}$. In other words these coreps may cause magnetic phase transitions. In Table III we show which coreps take one magnetic phase of $\mathrm{ZnO}$ to another magnetic phase of lower symmetry.

\section{Experimental}

In this section we discuss the possible experimental techniques for monitoring the magnetic transitions. $\mathrm{X}$-ray and cold neutron scattering are able to monitor the change of symmetry in crystals. To our best knowledge the experimental data on $\mathrm{ZnO}$ magnetic based on these two techniques are not available. The change of equilibrium positions of atoms or ions in crystals results in change of phonons states which can be deduced from the Raman spectroscopy. The states of phonons in magnetic crystals are classified according to relevant coreps. Therefore knowing the initial symmetry of the crystal and the symmetry after transitions the group theoretical methods are able to provide the active modes before and after transitions.

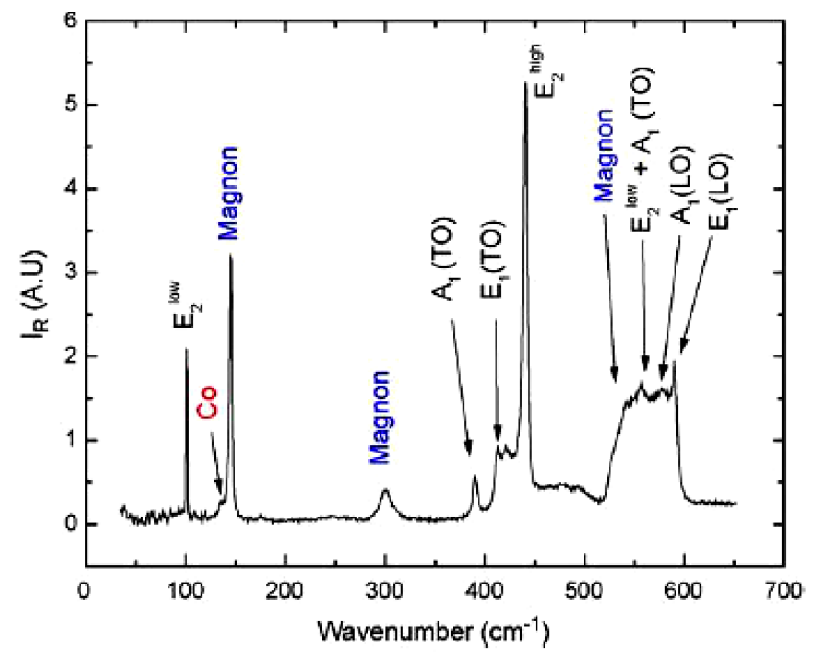

Fig. 1. Raman spectra of $\mathrm{ZnO}: \mathrm{Co}, A_{1} \rightarrow C \Gamma_{1}, E_{1} \rightarrow$ $C \Gamma_{3,5}, E_{2} \rightarrow C \Gamma_{4,6}$. M. Millot et. al. Journal of Alloys and compounds 423, 224 (2006).

In Fig. 1, the Raman spectrum of $\mathrm{ZnO}$ doped with $\mathrm{Co}$ shows the Raman active modes responsible for transition.

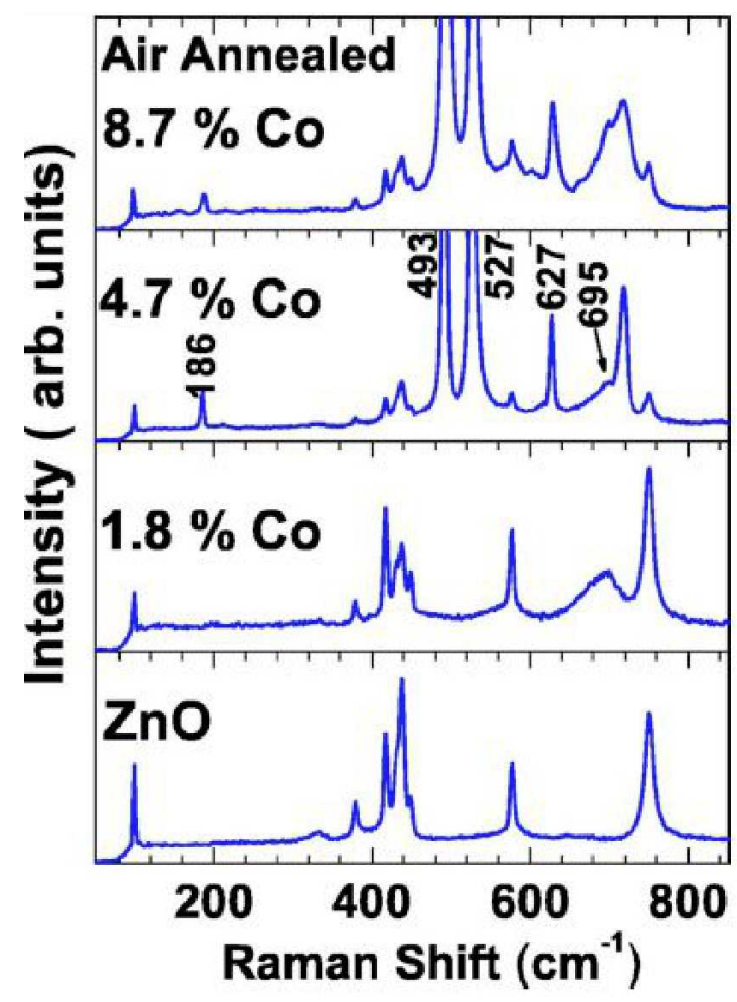

Fig. 2. Room temperature Raman spectra of air annealed $\mathrm{Co}_{x} \mathrm{Zn}_{1-x} \mathrm{O}$ films for different values of $x$. J.S. Thakur et al. Journal of Applied Physics 102, 093904 (2007).

The classification of the active modes in Fig. 1 is in terms of irreps. However the appropriate classification should be $A_{1} \rightarrow C \Gamma_{1}, E_{1} \rightarrow C \Gamma_{3,5}$ and $E_{2} \rightarrow C \Gamma_{4,6}$.

The Raman active modes are contained in symmetrized square of vector representation $[V]_{2}$ obtained from CDML [8]. Decomposing $[V]_{2}$ onto irreps and coreps of non-magnetic and magnetic space groups we obtain the Raman active modes of the crystal which appear in Fig. 1 and 2. For $\mathrm{ZnO}$, from the decomposition of $[V]_{2}$ we obtain the Raman active modes: $\Gamma_{1,5,6}\left(A_{1}, E_{1}, E_{2}\right)$ and for $\mathrm{ZnO}: \mathrm{Co}, C \Gamma_{1}, C \Gamma_{3,5}$ and $C \Gamma_{4,6}$.

Assigning phonons in Fig. 2, for air annealed and 8.7\% in the region (520-800) $\mathrm{cm}^{-1}$ by $C \Gamma_{1}, C \Gamma_{3,5}$ and $C \Gamma_{4,6}$ we may identify the coreps responsible for S.O.Ph.Tr and the magnetic subgroup after transition, $C_{6}^{6}\left(C_{3}^{1}\right)$ : $P 63^{\prime}(1,3,5, \theta 2.1, \theta 4.1, \theta 6.1)$. 


\section{Discussions and conclusion}

In this work, we investigate S.O.Ph.Tr in magnetic and non-magnetic $\mathrm{ZnO}$ based on $\mathrm{L}-\mathrm{L}$ and group theoretical methods. Our rigid calculations yield appropriate active irreps and coreps responsible for transitions. There are at least two ways to find appropriate subgroup of initial group $\mathrm{ZnO}$. Using our computational methods or CDML tables we find several possible space subgroups. Using Lyubarskii [2] we found subgroups caused by respective irreps and coreps. In other words irreps and coreps yield appropriate subgroups (structures of the crystals) appearing after S.O.Ph.Tr (see Tables II and III).

TABLE VI

Irreps of $\Gamma_{2}$ of $C_{6 v}^{4}$

\begin{tabular}{c|cccccccccccc}
\hline \hline & 1 & 2.1 & 3 & 4.1 & 5 & 6.1 & 19 & 20.1 & 21 & 22.1 & 23 & 24.1 \\
\hline$\Gamma_{2}$ & 1 & 1 & 1 & 1 & 1 & 1 & -1 & -1 & -1 & -1 & -1 & -1
\end{tabular}

For example from Table VI we see that only symmetry operators $1,2.1,3,4.1,5,6.1$ do not change the $\rho$ and belong to the subgroup $C_{6}^{6} / T$ of $C_{6 v}^{4} / T$. Similarly for the corep $\mathrm{CA}_{2}$ the following antiunitary operators, 1,3 , $5, \theta 19, \theta 21, \theta 23$ do not change the basis and they form the magnetic space subgroup $P 3 m^{\prime} 1$ of $P 6_{3} m^{\prime} c^{\prime}$ (see Table III).

To our best knowledge this is the first time of describing the theory of S.O.Ph.Tr using the classification of phonon states in magnetic crystals in terms of coreps. Previous descriptions of transitions in magnetic crystals were done using the classifications of phonons by ordinary unitary irreps. It is to be mentioned that this approach is not wrong but it is not fully satisfactory because the operator of time reversal symmetry is not taken into account. Clearly, the non-magnetic space group $C_{6 v}^{4}$ is a subgroup of $C_{6 v}^{4}$ magnetic with $P 6_{3}^{\prime} m^{\prime} c$ symmetry.

\section{References}

[1] L.D. Landau, E.M. Lifshitz, Statistical Physics, Pergamon Press, London 1958.

[2] G.Ya. Lyubarskii, The Application of Group Theory in Physics, translated by S. Dedijer, Pergamon Press, 1960.

[3] E.P. Wigner, Group Theory and its Application to the Quantum Mechanics of Atomic Spectra, Academic Press, New York 1959.

[4] A.P. Cracknell, Magnetism in Crystalline Materials, Pergamon Press, Oxford 1965.

[5] H.T. Stokes, D.M. Hatch, Phys. Rev. B 30, 4962 (1984).

[6] G. Fröbenius, I. Schur, Berl. Ber. 1906186 (1906).

[7] W.F. Karavaev, Sov. Phys.-Solid State 6, 2943 (1965).

[8] A.P. Cracknell, B.L. Davies, S.C. Miller, W.F. Love, Kronecker Product Tables, Vols. 1-4, IFI/Plenum, New York 1979. 